\title{
A mixed finite element method for elliptic optimal control problems using a three-field formulation
}

BP Lamichhane ${ }^{1} \quad$ A Kumar $^{2} \quad$ B Kalyanaraman ${ }^{3}$

Received 16 November 2017; revised 18 March 2018

\begin{abstract}
In this paper, we consider an optimal control problem governed by elliptic differential equations posed in a three-field formulation. Using the gradient as a new unknown we write a weak equation for the gradient using a Lagrange multiplier. We use a biorthogonal system to discretise the gradient, which leads to a very efficient numerical scheme. A numerical example is presented to demonstrate the convergence of the finite element approach.
\end{abstract}

Subject class: 49J20, 65L20

Keywords: Optimal control; biorthogonal system; a priori error estimates

DOI:10.21914/anziamj.v59i0.12643, (C) Austral. Mathematical Soc. 2018. Published July 3, 2018, as part of the Proceedings of the 13th Biennial Engineering Mathematics and Applications Conference. ISSN 1445-8810. (Print two pages per sheet of paper.) Copies of this article must not be made otherwise available on the internet; instead link directly to the DOI for this article. 


\section{Contents}

1 Introduction

C98

2 Finite element method

C101

3 Variational discretisation: error estimates

C104

4 Numerical results

C108

References

C109

\section{Introduction}

The cost functional of an optimal control problem governed by a partial differential equation often involves the solution as well as the gradient of the solution. In that situation, a better approximation of the gradient is obtained by using a mixed finite element method [1]. Recently, mixed finite element approaches have become quite popular to discretise optimal control problems involving elliptic partial differential equations [3-5].

In this paper, we apply a mixed finite element method to approximate the solution of an optimal control problem governed by a Poisson problem. In contrast to previous approaches [3-5], which are based on a two-field formulation of the Poisson problem, we use a three-field formulation of the Poisson problem which allows us to use a biorthogonal system in the discretisation. The use of a biorthogonal system allows us to statically condense out all the extra degrees of freedom we have in the mixed formulation leading to a very efficient finite element approach. The formulation is obtained by introducing the gradient of the solution of Poisson equation as a new unknown and writing an additional variational equation in terms of a Lagrange multiplier. An efficient numerical scheme is obtained by using a biorthogonal 
system to discretise the space of the gradient of the solution and the Lagrange multiplier space in the discrete setting.

Let $\Omega$ be a bounded and convex domain in $\mathbb{R}^{2}$ and $\Gamma$ the boundary of $\Omega$. In the following we use the usual notations for the Sobolev space $H^{k}(\Omega)$ for positive integer $k$, and the associated norm on $\mathrm{H}^{\mathrm{k}}(\Omega)$ with $\mathrm{L}^{2}(\Omega)=\mathrm{H}^{0}(\Omega)$ denoting the set of all real-valued square integrable functions defined on $\Omega$ [2]. Let $\alpha \in \mathbb{R}$ be a parameter, $y_{d} \in \mathrm{L}^{2}(\Omega)$ the desired state and $\sigma_{d} \in\left[\operatorname{L}^{2}(\Omega)\right]^{2}$ the desired gradient. Consider the following optimal control problem

$$
\min _{\mathfrak{u} \in \mathrm{U}_{\mathrm{ad}}} \mathrm{J}(\mathrm{y}, \mathfrak{u}):=\frac{1}{2}\left\|\mathrm{y}-\mathrm{y}_{\mathrm{d}}\right\|_{0, \Omega}^{2}+\frac{1}{2}\left\|\sigma-\sigma_{\mathrm{d}}\right\|_{0, \Omega}^{2}+\frac{\alpha}{2}\|\mathfrak{u}\|_{0, \Omega}^{2}, \quad \sigma=\nabla \mathbf{y},
$$

subject to the partial differential equation

$$
-\nabla \cdot \mathrm{K} \nabla \mathrm{y}=\mathrm{Bu}+\mathrm{f} \quad \text { in } \Omega \text { with } \mathrm{y}=0 \quad \text { on } \Gamma,
$$

where $\mathrm{K} \in \mathrm{L}^{\infty}\left(\Omega, \mathbb{R}^{2 \times 2}\right)$ is a real-valued, symmetric and positive definite matrix, and $\mathrm{B}$ is the bounded linear operator defined on the set of admissible set of controls $\mathrm{U}_{\mathrm{ad}}$

$$
\mathrm{u}_{\mathrm{ad}}=\left\{\mathrm{u} \in \mathrm{L}^{2}(\Omega):\|\mathrm{u}\|_{0, \Omega} \leqslant \mathrm{M}\right\},
$$

so that for some positive constant $b$

$$
\|\mathrm{Bu}\|_{0, \Omega} \leqslant \mathrm{b}\|\mathrm{u}\|_{0, \Omega}, \quad \mathfrak{u} \in \mathrm{U}_{\mathrm{ad}} .
$$

The existence and uniqueness of the optimal control follows from the strict convexity [13]. Using $\mathrm{B}^{*}$ as the adjoint operator of B the first order optimality condition can be formulated as

$$
\left(\alpha u+B^{*} p, v-u\right) \geqslant 0, \quad v \in U_{a d},
$$

where $p$ is the adjoint state associated with $u$ and it solves the following adjoint equation of finding $\mathrm{p} \in \mathrm{H}_{0}^{1}(\Omega)$ such that

$$
\int_{\Omega}\left[K \nabla p \cdot \nabla q+\left(\nabla y-\sigma_{d}\right) \cdot \nabla q+\left(y-y_{d}\right) q\right] d x=0, \quad q \in H_{0}^{1}(\Omega) .
$$


The variational inequality in equation (3) can be written as

$$
u(x)=P_{[-M, M]}\left(-\frac{B^{*} p}{\alpha}\right),
$$

where $[12,13]$

$$
P_{[a, b]}(f(x))=\max (a, \min (b, f(x))) .
$$

We start with the following variational form of (2) to recast our problem as a three-field formulation:

$$
\min _{y \in H_{0}^{1}(\Omega)} \frac{1}{2} \int_{\Omega} K \nabla y \cdot \nabla y d x-\int_{\Omega}(f+B u) y d x,
$$

and introduce the gradient of the state of the system as a new unknown $\sigma=\nabla \mathbf{y}$, and write its weak equation as

$$
\int_{\Omega}(\sigma-\nabla \mathrm{y}) \cdot \psi \mathrm{dx}=0, \quad \psi \in\left[\mathrm{L}^{2}(\Omega)\right]^{2},
$$

where $\psi$ acts as a Lagrange multiplier. Using $V=H_{0}^{1}(\Omega), R=\left[\mathrm{L}^{2}(\Omega)\right]^{2}$, and $\ell(y)=\int_{\Omega}(f+B u) y d x$, we get a constrained minimisation problem, which leads to the following saddle point problem of finding $(y, \sigma, \phi) \in V \times R \times R$ such that

$$
\begin{aligned}
\tilde{a}((y, \sigma),(z, \tau))+\quad b((z, \tau), \phi) & =\ell(z), & (z, \tau) \in \mathrm{V} \times \mathrm{R}, \\
\mathrm{b}((y, \sigma), \psi) & =0, & \psi \in R,
\end{aligned}
$$

where

$$
\tilde{a}((y, \sigma),(z, \tau))=\int_{\Omega} \mathrm{K} \sigma \cdot \tau \mathrm{d} x, \quad \mathrm{~b}((y, \sigma), \psi)=\int_{\Omega}(\sigma-\nabla y) \cdot \psi d x .
$$

Thus our optimal control problem is to find $(\mathrm{y}, \sigma, \phi, \mathfrak{u}) \in \mathrm{V} \times \mathrm{R} \times \mathrm{R} \times \mathrm{U}_{\mathrm{ad}}$ such that (6) is satisfied as well as the inequality (3), where $p=\tilde{y}$ in (3) is 
one field in the problem of finding three fields $(\tilde{y}, \tilde{\sigma}, \tilde{\phi}) \in \mathrm{V} \times \mathrm{R} \times \mathrm{R}$ such that

$$
\begin{aligned}
\int_{\Omega} \tilde{\phi} \cdot \nabla z d x-\int_{\Omega}\left(y-y_{d}\right) z d x=0, & z \in V, \\
\int_{\Omega}(K \tilde{\sigma}+\tilde{\phi}) \cdot \tau d x+\int_{\Omega}\left(\sigma-\sigma_{d}\right) \cdot \tau d x=0, & \tau \in R, \\
\int_{\Omega}(\tilde{\sigma}-\nabla \tilde{y}) \cdot \psi d x=0, & \psi \in R .
\end{aligned}
$$

Here the adjoint equations are derived from the Euler-Lagrange equations of the following minimisation problem

$$
\underset{(\tilde{y}, \tilde{\sigma}) \in[\mathbf{V} \times \mathrm{R}]}{\arg \min }\left(\frac{1}{2} \int_{\Omega} \mathrm{K} \tilde{\sigma} \cdot \tilde{\sigma} \mathrm{d} x-\tilde{\ell}(\tilde{\mathbf{y}}, \tilde{\sigma})\right),
$$

under the constraint

$$
\int_{\Omega}(\tilde{\sigma}-\nabla \tilde{y}) \cdot \psi d x=0, \quad \psi \in R
$$

where

$$
\tilde{\ell}(\tilde{y}, \tilde{\sigma})=\int_{\Omega}\left(y_{d}-y\right) \tilde{y} d x-\int_{\Omega}\left(\sigma-\sigma_{d}\right) \cdot \tilde{\sigma} d x .
$$

To get a stable discrete formulation we replace the bilinear form $\tilde{a}(\cdot, \cdot)$ by the bilinear form $a(\cdot, \cdot)[6]$ defined as

$$
a((y, \sigma),(z, \tau))=\frac{1}{2} \int_{\Omega}(K \sigma \cdot \tau+K \nabla y \cdot \nabla z) d x .
$$

\section{Finite element method}

Let $\mathcal{T}_{h}$ be a quasi-uniform partition of the domain $\Omega$ in triangles. We use the standard linear finite element space on the mesh $\mathcal{T}_{h}$ defined as

$$
S_{\mathrm{h}}=\left\{z_{\mathrm{h}} \in \mathrm{H}^{1}(\Omega):\left.z_{\mathrm{h}}\right|_{\mathrm{T}} \in \mathcal{P}_{1}(\mathrm{~T}), \mathrm{T} \in \mathcal{T}_{\mathrm{h}}\right\}, \quad \mathrm{V}_{\mathrm{h}}=\mathrm{S}_{\mathrm{h}} \cap \mathrm{H}_{0}^{1}(\Omega),
$$


where $\mathcal{P}_{1}(T)$ is the space of linear polynomials in $T$ [2].

Let $\left\{\varphi_{1}, \varphi_{2}, \cdots, \varphi_{\mathrm{N}}\right\}$ be the finite element basis for $S_{\mathrm{h}}$. We now construct a set of basis functions $\left\{\mu_{1}, \mu_{2}, \cdots, \mu_{N}\right\}$ of another finite element space $W_{h}$ so that the basis functions of $S_{h}$ and $W_{h}$ satisfy a condition of biorthogonality relation

$$
\int_{\Omega} \varphi_{i} \mu_{j} d x=c_{j} \delta_{i j}, \quad c_{j} \neq 0, \quad 1 \leqslant i, j, \leqslant N,
$$

where $\delta_{i j}$ is the Kronecker symbol, and $c_{j}$ a scaling factor [10]. Hence the sets of basis functions of $S_{h}$ and $W_{h}$ form a biorthogonal system. The finite element space for the gradient of the solution is

$$
\mathrm{L}_{\mathrm{h}}=\left[\mathrm{S}_{\mathrm{h}}\right]^{2},
$$

and for the Lagrange multiplier is

$$
M_{h}=\left[W_{h}\right]^{2} .
$$

Our discrete problem is to find $\left(y_{h}, \sigma_{h}, \varphi_{h}\right) \in V_{h} \times L_{h} \times M_{h}$ such that

$$
\begin{array}{llrl}
\mathrm{a}\left(\left(y_{h}, \sigma_{h}\right),\left(z_{h}, \tau_{h}\right)\right)+\mathrm{b}\left(\left(z_{h}, \tau_{h}\right), \varphi_{h}\right) & =\ell\left(z_{h}\right), & \left(z_{h}, \tau_{h}\right) & \in V_{h} \times L_{h} \\
b\left(\left(y_{h}, \sigma_{h}\right), \psi_{h}\right) & =0, & \psi_{h} & \in M_{h},
\end{array}
$$

We now introduce a projection operator $\mathrm{Q}_{h}: \mathrm{L}^{2}(\Omega) \rightarrow S_{h}$ defined as

$$
\int_{\Omega} Q_{h} v \mu_{h} d x=\int_{\Omega} v \mu_{h} d x, \quad \mu_{h} \in W_{h}, v \in L^{2}(\Omega) .
$$

Due to the biorthogonality relation (11), $\mathrm{Q}_{\mathrm{h}}$ is well-defined, and $\mathrm{Q}_{\mathrm{h}} \boldsymbol{v}$ for $v \in \mathrm{L}^{2}(\Omega)$ is given by

$$
\mathrm{Q}_{h} v=\sum_{i=1}^{n} \frac{\int_{\Omega} \mu_{\mathrm{i}} v d x}{c_{i}} \varphi_{i}
$$

Using the notation that $\mathrm{Q}_{\mathrm{h}}$ is applied component-wise when applied to a vector function the second equation of (12) leads to $\sigma_{h}=Q_{h}\left(\nabla y_{h}\right)$. Hence 
static condensation of the gradient of the solution and the Lagrange multiplier leads to the following problem of finding $y_{h} \in V_{h}$ such that

$$
A_{h}\left(y_{h}, z_{h}\right)=\ell\left(z_{h}\right), \quad z_{h} \in V_{h},
$$

where

$$
A_{h}\left(y_{h}, z_{h}\right)=\frac{1}{2}\left(\int_{\Omega} K Q_{h} \nabla y_{h} \cdot Q_{h} \nabla z_{h} d x+\int_{\Omega} K \nabla y_{h} \cdot \nabla z_{h} d x\right) .
$$

Using the same approach to discretise the adjoint equations (7) - (9) we have the problem of finding $\left(\tilde{y}_{h}, \tilde{\sigma}_{h}, \tilde{\phi}_{h}\right) \in V_{h} \times L_{h} \times M_{h}$ such that

$$
\begin{aligned}
\int_{\Omega} \tilde{\phi}_{h} \cdot \nabla z_{h} d x-\int_{\Omega}\left(y_{h}-y_{d}\right) z_{h} d x=0, & z_{h} \in V_{h}, \\
\int_{\Omega}\left(K \tilde{\sigma}_{h}+\tilde{\phi}_{h}\right) \cdot \tau_{h} d x+\int_{\Omega}\left(\sigma_{h}-\sigma_{d}\right) \cdot \tau_{h} d x=0, & \tau_{h} \in L_{h}, \\
\int_{\Omega}\left(\tilde{\sigma}_{h}-\nabla \tilde{y}_{h}\right) \cdot \psi_{h} d x=0, & \psi \in M_{h} .
\end{aligned}
$$

We note that (15) leads to $\tilde{\sigma}_{h}=Q_{h} \nabla \tilde{y}_{h}$. Using this and setting $p_{h}=\tilde{y}_{h}$, we arrive at the problem of finding $p_{h} \in V_{h}$ such that for all $q_{h} \in V_{h}$ we have

$$
\begin{aligned}
\int_{\Omega}\left(K Q_{h} \nabla p_{h}\right) \cdot\left(Q_{h} \nabla q_{h}\right) d x= & \int_{\Omega}\left(y_{d}-y_{h}\right) q_{h} d x \\
& -\int_{\Omega}\left(Q_{h} \nabla y_{h}-\sigma_{d}\right) \cdot\left(Q_{h} \nabla q_{h}\right) d x .
\end{aligned}
$$

Using the same stabilisation approach as for the state variable, we now obtain the equation of finding $p_{h} \in V_{h}$ such that

$$
A_{h}\left(p_{h}, q_{h}\right)=\int_{\Omega}\left(y_{d}-y_{h}\right) q_{h} d x-\int_{\Omega}\left(Q_{h} \nabla y_{h}-\sigma_{d}\right) \cdot\left(Q_{h} \nabla q_{h}\right) d x, q_{h} \in V_{h} .
$$




\section{Variational discretisation: error estimates}

Now we consider the discrete formulation of the state and adjoint state equations where the control $u$ is not discretized initially but given by a projection formula $(18)[12,13]$. The discrete problem is then to find $\left(\mathfrak{y}_{h}, p_{h}, \mathfrak{u}_{h}\right) \in$ $V_{h} \times V_{h} \times U_{a d}$ such that

$$
\begin{aligned}
A_{h}\left(y_{h}, z_{h}\right) & =\left(B u_{h}+f, z_{h}\right), \quad z_{h} \in V_{h}, \\
A_{h}\left(p_{h}, q_{h}\right) & =\left(y_{d}-y_{h}, q_{h}\right)-\left(Q_{h} \nabla y_{h}-\sigma_{d}, Q_{h} \nabla q_{h}\right), \quad q_{h} \in V_{h},(17) \\
u_{h} & =-P_{[a, b]}\left(\frac{B^{*} p_{h}}{\alpha}\right) .
\end{aligned}
$$

where the bilinear form $A_{h}(\cdot, \cdot)$ satisfies the following continuity and coercivity properties with respect to the $\mathrm{H}^{1}$-norm for two positive constants $\alpha$ and $\beta$ independent of $h$ :

(i) $A_{h}\left(\phi_{h}, \psi_{h}\right) \leqslant \alpha\left\|\phi_{h}\right\|_{1, \Omega}\left\|\psi_{h}\right\|_{1, \Omega}, \quad \phi_{h}, \psi_{h} \in V_{h}$.

(ii) $A_{h}\left(\phi_{h}, \phi_{h}\right) \geqslant \beta\left\|\phi_{h}\right\|_{1, \Omega}^{2}, \quad \phi_{h} \in V_{h}$.

Since $\mathrm{Q}_{\mathrm{h}}$ is stable in $\mathrm{H}^{1}$ and $\mathrm{L}^{2}$-norms [10], and $\mathrm{B}$ is bounded, the right hand sides of both variational equations (16) and (17) are continuous. Thus both variational equations (16) and (17) have unique solutions by Lax-Milgram lemma. We note that the variational inequality

$$
\left(\alpha u_{h}+B^{*} p_{h}, v-u_{h}\right) \geqslant 0, \quad v \in u_{a d}
$$

for the control $\mathfrak{u}_{\mathfrak{h}}$ is replaced by the projection formula (18).

For a fixed $u$, let $\boldsymbol{y}_{\mathfrak{h}}(\mathfrak{u})$ be the solution of

$$
A_{h}\left(y_{h}(u), z_{h}\right)=\left(B u+f, z_{h}\right), \quad z_{h} \in V_{h},
$$

and for a fixed $y$, let $p_{h}(y)$ be the solution of

$$
A_{h}\left(p_{h}(y), q_{h}\right)=\left(y_{d}-y, q_{h}\right)-\left(Q_{h} \nabla y-\sigma_{d}, Q_{h} \nabla q_{h}\right), \quad q_{h} \in V_{h}(20)
$$


Then under the assumption that the domain $\Omega$ is convex, we have the following approximation results for the solutions of the discrete equations (19) and (20).

Theorem 1. Let $\mathrm{y}_{\mathrm{h}}(\mathrm{u})$ and $\mathrm{p}_{\mathrm{h}}(\mathrm{y})$ be the solutions of (19) and (20) respectively. Then there exists a constant $\mathrm{C}$, independent of $\mathrm{h}$, such that

$$
\begin{aligned}
& \left\|y-y_{h}(u)\right\|_{0, \Omega}+h\left\|y-y_{h}(u)\right\|_{1, \Omega} \leqslant \mathrm{Ch}^{2}\|y\|_{2, \Omega} \\
& \left\|p-p_{h}(y)\right\|_{0, \Omega}+h\left\|p-p_{h}(y)\right\|_{1, \Omega} \leqslant \mathrm{Ch}^{2}\|p\|_{2, \Omega} .
\end{aligned}
$$

Setting $y_{h}=y_{h}\left(u_{h}\right)$ and $p_{h}=p_{h}\left(y_{h}\right)$, we have the following result.

Lemma 2. Let $\mathrm{y}_{\mathrm{h}}(\mathrm{u})$ and $\mathrm{p}_{\mathrm{h}}(\mathrm{y})$ be the solutions of (19) and (20) respectively. Then there exists a constant $\mathrm{C}$, independent of $\mathrm{h}$, such that

$$
\begin{aligned}
& \left\|y_{h}(u)-y_{h}\right\|_{1, \Omega} \leqslant C\left\|u-u_{h}\right\|_{0, \Omega} \\
& \left\|p_{h}(y)-p_{h}\right\|_{1, \Omega} \leqslant C\left\|y-y_{h}\right\|_{0, \Omega} .
\end{aligned}
$$

The proof of this lemma follows from the coercivity of $A_{h}(\cdot, \cdot)$ [11, Lemma 3.1].

Theorem 3. Let $(\mathrm{y}, \mathrm{p}, \mathrm{u}) \in\left(\mathrm{H}^{2}(\Omega) \cap \mathrm{H}_{0}^{1}(\Omega)\right) \times\left(\mathrm{H}^{2}(\Omega) \cap \mathrm{H}_{0}^{1}(\Omega)\right) \times \mathrm{U}_{\mathrm{ad}}$ be the solutions of (1) and (2) with $\mathrm{u} \in \mathrm{H}^{1}(\Omega)$, and $\left(\mathrm{y}_{\mathrm{h}}, \mathrm{p}_{\mathrm{h}}, \mathrm{u}_{\mathrm{h}}\right) \in \mathrm{V}_{\mathrm{h}} \times \mathrm{V}_{\mathrm{h}} \times \mathrm{U}_{\mathrm{ad}}$ be the solutions of (16)-(18). Then for sufficiently small $\mathrm{h}$ there exists a mesh-independent constant $\mathrm{C}$ such that

$$
\left\|\mathrm{u}-\mathrm{u}_{\mathrm{h}}\right\|_{0, \Omega} \leqslant \mathrm{Ch}, \quad\left\|\mathrm{y}-\mathrm{y}_{\mathrm{h}}\right\|_{0, \Omega} \leqslant \mathrm{Ch}, \quad\left\|\mathrm{p}-\mathrm{p}_{\mathrm{h}}\right\|_{0, \Omega} \leqslant \mathrm{Ch} .
$$

Proof: From the optimality condition, we get

$$
\begin{aligned}
\alpha\left\|u-u_{h}\right\|_{0, \Omega}^{2} & \leqslant\left(p-p_{h}, B\left(u_{h}-u\right)\right) \\
& =\left(p-p_{h}(y), B\left(u_{h}-u\right)\right)+\left(p_{h}(y)-p_{h}, B\left(u_{h}-u\right)\right) .
\end{aligned}
$$

From (19), we have

$$
A_{h}\left(y_{h}-y_{h}(u), z_{h}\right)=\left(B\left(u_{h}-u\right), z_{h}\right) .
$$


Setting $z_{h}=p_{h}(y)-p_{h}$ we get from the above equation

$$
A_{h}\left(y_{h}-y_{h}(u), p_{h}(y)-p_{h}\right)=\left(B\left(u_{h}-u\right), p_{h}(y)-p_{h}\right) .
$$

Thus

$$
\begin{aligned}
\alpha\left\|u-u_{h}\right\|_{0, \Omega}^{2} \leqslant & \left(p-p_{h}(y), B\left(u_{h}-u\right)\right) \\
& +A_{h}\left(p_{h}(y)-p_{h}, y_{h}-y_{h}(u)\right) .
\end{aligned}
$$

From (20), we get

$$
A_{h}\left(p_{h}(y)-p_{h}, q_{h}\right)=\left(y_{h}-y, q_{h}\right)-\left(Q_{h}\left(\nabla y-\nabla y_{h}\right), Q_{h} \nabla q_{h}\right) .
$$

We now use $q_{h}=y_{h}(u)-y_{h}$ in the above equation to write

$$
\begin{aligned}
& A_{h}\left(p_{h}(y)-p_{h}, y_{h}(u)-y_{h}\right) \\
= & \left(y_{h}-y, y_{h}(u)-y_{h}\right)-\left(Q_{h} \nabla\left(y-y_{h}\right), Q_{h} \nabla\left(y_{h}(u)-y_{h}\right)\right) \\
= & \left(y_{h}(u)-y, y_{h}(u)-y_{h}\right)-\left(y_{h}(u)-y_{h}, y_{h}(u)-y_{h}\right) \\
& -\left(Q_{h} \nabla\left(y-y_{h}(u)\right), Q_{h} \nabla\left(y_{h}(u)-y_{h}\right)\right) \\
& +\left(Q_{h} \nabla\left(y_{h}-y_{h}(u)\right), Q_{h} \nabla\left(y_{h}(u)-y_{h}\right)\right) \\
= & \left(y_{h}(u)-y, y_{h}-y_{h}(u)\right)-\left\|y_{h}(u)-y_{h}\right\|_{0, \Omega}^{2} \\
& +\left(Q_{h} \nabla\left(y-y_{h}(u)\right), Q_{h} \nabla\left(y_{h}-y_{h}(u)\right)\right)-\| Q_{h} \nabla\left(y_{h}-y_{h}(u) \|_{0, \Omega}^{2}\right. \\
\leqslant & C\left\|y-y_{h}(u)\right\|_{1, \Omega}\left\|y_{h}-y_{h}(u)\right\|_{1, \Omega} \\
\leqslant & C h\|y\|_{2, \Omega}\left\|u-u_{h}\right\|_{0, \Omega},
\end{aligned}
$$

where we use the $\mathrm{L}^{2}$ stability of $\mathrm{Q}_{\mathrm{h}}$, Theorem 1 and Lemma 2. Now we use the above estimate for the second term on the right of (24) and use the following estimate for the first term on the right of (24)

$$
\left|\left(p-p_{h}(y), B\left(u_{h}-\mathfrak{u}\right)\right)\right| \leqslant C h^{2}\left\|u-u_{h}\right\|_{0, \Omega}\|p\|_{2, \Omega},
$$

to write (24) as

$$
\alpha\left\|u-u_{h}\right\|_{0, \Omega}^{2} \leqslant \mathrm{Ch}^{2}\|p\|_{2, \Omega}\left\|u-u_{h}\right\|_{0, \Omega}+C h\|y\|_{2, \Omega}\left\|u-u_{h}\right\|_{0, \Omega},
$$


which yields

$$
\left\|\mathfrak{u}-\mathfrak{u}_{\mathrm{h}}\right\|_{0, \Omega} \leqslant \mathrm{C}\left(\mathrm{h}^{2}\|\mathrm{p}\|_{2, \Omega}+\mathrm{h}\|\mathrm{y}\|_{2, \Omega}\right) .
$$

In order to estimate $\left\|\mathrm{y}-\mathbf{y}_{\mathbf{h}}\right\|_{0, \Omega}$, we start with

$$
\left\|y-y_{h}\right\|_{0, \Omega} \leqslant\left\|y-y_{h}(u)\right\|_{0, \Omega}+\left\|y_{h}(u)-y_{h}\right\|_{0, \Omega} .
$$

For the first term on the right of the above estimate we have $\left\|y-y_{h}(u)\right\|_{0, \Omega} \leqslant$ $\mathrm{Ch}^{2}\|\mathrm{y}\|_{2, \Omega}$, and for the second term on the right we use Lemma 2 to write

$$
\left\|y_{h}(u)-y_{h}\right\|_{0, \Omega} \leqslant C\left\|y_{h}(u)-y_{h}\right\|_{1, \Omega} \leqslant C\left\|u-u_{h}\right\|_{0, \Omega} .
$$

Now the result follows using the estimate for $\left\|\mathfrak{u}-\mathfrak{u}_{\mathrm{h}}\right\|_{0, \Omega}$. Similarly, to estimate $\left\|p-p_{h}\right\|_{0, \Omega}$, we use the triangle inequality and write

$$
\left\|p-p_{h}\right\|_{0, \Omega} \leqslant\left\|p-p_{h}(y)\right\|_{0, \Omega}+\left\|p_{h}(y)-p_{h}\right\|_{0, \Omega} .
$$

Since $\left\|\mathrm{p}-\mathrm{p}_{\mathrm{h}}(\mathrm{y})\right\|_{0, \Omega} \leqslant \mathrm{Ch}^{2}\|\mathrm{p}\|_{2, \Omega}$, we estimate the second term on the right side of the above estimate by using Lemma 2 to get

$$
\left\|p_{h}(y)-p_{h}\right\|_{0, \Omega} \leqslant C\left\|p_{h}(y)-p_{h}\right\|_{1, \Omega} \leqslant C\left\|y-y_{h}\right\|_{0, \Omega} .
$$

The final result follows on using the estimate for $\left\|y-y_{h}\right\|_{0, \Omega}$.

Remark 4. Using the standard saddle point theory [1] we have the following error estimate for the error in the gradient $\sigma=\nabla y$ :

$$
\left\|\sigma-\sigma_{h}\right\|_{0, \Omega} \leqslant \mathrm{Ch}
$$

where $\sigma_{h}=Q_{h} \nabla y_{h}$ with $y$ and $y_{h}$ as defined in the above theorem.

Remark 5. The approach based on a biorthogonal system can be easily extended to a three-dimensional problem and other elliptic partial differential equations [7-9]. 

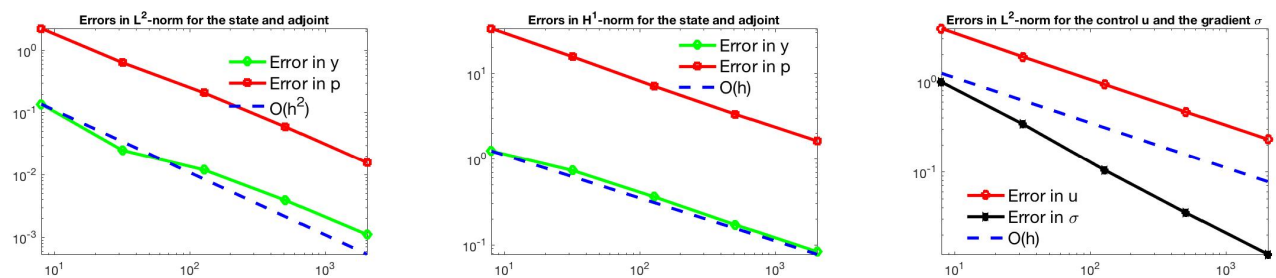

Figure 1: $L^{2}$ errors for the state $y$ and adjoint $p$ (left), $H^{1}$ errors for the state $y$ and the adjoint $p$ (middle) and $\mathrm{L}^{2}$ errors for the control $u$ and the gradient $\sigma$ (right) versus the number of elements

\section{Numerical results}

In this section, we present a numerical example to support the error estimates proved in the last section. We have used the primal dual active set strategy to compute the solution of the discrete formulation [13]. Let $\Omega=[0,1]^{2}$ with the boundary $\Gamma$. We consider the following problem

$$
\min _{\mathfrak{u} \in \mathrm{U}_{\mathrm{ad}}} \mathrm{J}(\mathrm{y}, \mathfrak{u})=\frac{1}{2}\left\|\mathrm{y}-\mathrm{y}_{\mathrm{d}}\right\|_{0, \Omega}^{2}+\frac{1}{2}\left\|\sigma-\sigma_{\mathrm{d}}\right\|_{0, \Omega}^{2}+\frac{1}{2}\|\mathfrak{u}\|_{0, \Omega}^{2}
$$

subject to

$$
-\Delta y=u \quad \text { in } \Omega \quad \text { with } y=0 \quad \text { in } \Gamma,
$$

where the exact solution for the state $y=\sin \left(\pi x_{1}\right) \sin \left(\pi x_{2}\right)$, the adjoint state $p=2 \pi^{2} \sin \left(\pi x_{1}\right) \sin \left(\pi x_{2}\right)$, and the control $u=\max (a, \min (b, p))$ with $\mathrm{a}=-25, \mathrm{~b}=25$. The desired states $\mathrm{y}_{\mathrm{d}}$ and $\sigma_{\mathrm{d}}$ are taken as

$$
\begin{aligned}
& y_{\mathrm{d}}=\sin \left(\pi x_{1}\right) \sin \left(\pi x_{2}\right), \\
& \sigma_{\mathrm{d}}=\left[\left(\pi+2 \pi^{3}\right) \cos \left(\pi x_{1}\right) \sin \left(\pi x_{2}\right),\left(\pi+2 \pi^{3}\right) \sin \left(\pi x_{1}\right) \cos \left(\pi x_{2}\right)\right] .
\end{aligned}
$$

We have shown the errors for the state $y$, adjoint state $p$, the control $u$ and the gradient $\sigma$ in Figure 1. We can see the convergence rates as predicted by the theory. The convergence rates for the gradient $\sigma$ are very close to 1.5 , which is better than the expected rate. 


\section{References}

[1] D. Boffi, F. Brezzi, and M. Fortin. Mixed finite element methods and applications. Springer-Verlag, 2013. doi:10.1007/978-3-642-36519-5. C98, C107

[2] S.C. Brenner and L.R. Scott. The Mathematical Theory of Finite Element Methods. Springer-Verlag, New York, 1994. doi:10.1007/978-0-387-75934-0. C99, C102

[3] Yanping Chen. Superconvergence of quadratic optimal control problems by triangular mixed finite element methods. International journal for numerical methods in engineering, 75(8):881-898, 2008. doi:10.1002/nme.2272. C98

[4] Hongfei Fu, Hongxing Rui, Jian Hou, and Haihong Li. A stabilized mixed finite element method for elliptic optimal control problems. Journal of Scientific Computing, 66(3):968-986, 2016. doi:10.1007/s10915-015-0050-3. C98

[5] Hui Guo, Hongfei Fu, and Jiansong Zhang. A splitting positive definite mixed finite element method for elliptic optimal control problem. Applied Mathematics and Computation, 219(24):11178-11190, August 2013. doi:10.1016/j.amc.2013.05.020. C98

[6] Muhammad Ilyas and Bishnu P. Lamichhane. A stabilised mixed finite element method for the poisson problem based on a three-field formulation. In M. Nelson, D. Mallet, B. Pincombe, and J. Bunder, editors, Proceedings of EMAC-2015, volume 57 of ANZIAM J., pages C177-C192. Cambridge University Press, 2016. doi:10.21914/anziamj.v57i0.10356. C101

[7] Bishnu P Lamichhane, AT McBride, and BD Reddy. A finite element method for a three-field formulation of linear elasticity based on 
biorthogonal systems. Computer Methods in Applied Mechanics and Engineering, 258:109-117, 2013. doi:10.1016/j.cma.2013.02.008. C107

[8] B.P. Lamichhane. Inf-sup stable finite element pairs based on dual meshes and bases for nearly incompressible elasticity. IMA Journal of Numerical Analysis, 29:404-420, 2009. doi:10.1093/imanum/drn013. C107

[9] B.P. Lamichhane. A mixed finite element method for the biharmonic problem using biorthogonal or quasi-biorthogonal systems. Journal of Scientific Computing, 46:379-396, 2011. doi:10.1007/s10915-010-9409-7. C107

[10] B.P. Lamichhane and E. Stephan. A symmetric mixed finite element method for nearly incompressible elasticity based on biorthogonal systems. Numerical Methods for Partial Differential Equations, 28:1336-1353, 2012. doi:10.1002/num.20683. C102, C104

[11] Xianbing Luo, Yanping Chen, and Yunqing Huang. Some error estimates of finite volume element approximation for elliptic optimal control problems. International Journal of Numerical Analysis and Modeling, 10(3):697-711, 2013. http://www.math.ualberta.ca/ ijnam/Volume-10-2013/No-3-13/2013-03-11.pdf. C105

[12] Fredi Tröltzsch. On finite element error estimates for optimal control problems with elliptic PDEs. In International Conference on Large-Scale Scientific Computing, pages 40-53. Springer, 2009. doi:10.1007/978-3-642-12535 - 54. C100, C104

[13] Fredi Tröltzsch. Optimal control of partial differential equations, volume 112. American Mathematical Society, 2010.

http://www. ams.org/books/gsm/112/. C99, C100, C104, C108 


\section{Author addresses}

1. BP Lamichhane, School of Mathematical \& Physical Sciences, University of Newcastle, Callaghan, NSW 2308, Australia mailto:Bishnu.Lamichhane@newcastle.edu.au

2. A Kumar, Department of Mathematics, BITS Pilani, KK Birla Goa Campus, Goa (India) 403726

mailto:anilpundir@goa.bits-pilani.ac.in

3. B Kalyanaraman, School of Mathematical \& Physical Sciences, University of Newcastle, Callaghan, NSW 2308, Australia mailto:balaje6@gmail.com 